\title{
Review
}

\section{The aliens inside human DNA: HERV-W/MSRV/syncytin-1 endogenous retroviruses and neurodegeneration}

\author{
Antonina Dolei, Elena Uleri, Gabriele Ibba, Maurizio Caocci, Claudia Piu, Caterina Serra \\ Department of Biomedical Sciences, University of Sassari, Sassari, Italy
}

\begin{abstract}
The human genome contains remnants of ancestral retroviruses now endogenously transmitted, called human endogenous retroviruses (HERVs). HERVs can be variably expressed, and both beneficial and detrimental effects have described. This review focuses on the MSRV and syncytin-1 HERV-W elements in relationship to neurodegeneration in view of their neuro-pathogenic and immune-pathogenic properties. Multiple sclerosis (MS) and a neurodegenerative disease (neuroAIDS) are reported in this review. In vivo studies in patients and controls for molecular epidemiology and follow-up studies are reviewed, along with in vitro cellular studies of the effects of treatments and of molecular mechanisms.

HERV-W/MSRV has been repeatedly found in MS patients (in blood, spinal fluid, and brain samples), and MRSV presence/load strikingly parallels MS stages and active/remission phases, as well as therapy outcome. The DNA of MS patients has increased MSRVenv copies, while syncytin-1 copies are unchanged in controls. Presence of MSRV in the spinal fluid predicted the worst MS progression, ten years in advance. The Epstein-Barr virus (EBV) activates HERV-W/MSRV both in vitro and in vivo. With respect to neuroAIDS, the HIV transactivator of transcription (Tat) protein activates HERV-W/MSRV in monocytes/macrophages and astrocytes indirectly by interaction with TLR4 and induction of TNF $\alpha$.

HERV-W/MSRV can be considered a biomarker for MS behavior and therapy outcome. Regarding MS pathogenesis, we postulate the possibility for EBV of an initial trigger of future MS, years later, and for MSRV of a direct role of effector of neuropathogenesis during MS. Additionally, HERV-W/MSR/syncytin-1 activation by HIV Tat could contribute to the HIV-related neurodegeneration.
\end{abstract}

Key words: endogenous retroviruses; HERV-W/MSRV/syncytin-1; multiple sclerosis; neuroAIDS.

J Infect Dev Ctries 2015; 9(6):577-587. doi:10.3855/jidc.6916

(Received 25 March 2015 - Accepted 21 May 2015)

Copyright $\odot 2015$ Dolei et al. This is an open-access article distributed under the Creative Commons Attribution License, which permits unrestricted use, distribution, and reproduction in any medium, provided the original work is properly cited.

\section{Introduction}

Human DNA contains a large amount of "alien" material, since an average of $8 \%$ of all our DNA is of retroviral origin [1]. It is well known that retrovirus replication requires provirus integration into the host DNA and that the integrated provirus becomes functionally indistinguishable from host sequences, behaving thereafter as a heritable cellular gene transmitted to progeny cells. If the progeny cells are germline cells, this endogenous infection can be transmitted vertically through generations. This process is ongoing in koalas, where the koala retrovirus, which is associated with neoplasias, shows features of transition between an exogenous and endogenous vertically transmitted element [2-3]. This phenomenon occurred repeatedly in our progenitors, with increasing loss, damage, reinfections, and rearrangements of the sequences. Nevertheless, similar to other animal species, the human genome contains remnants of many different kinds of retroviruses that are now endogenously transmitted and are hence called human endogenous retroviruses (HERVs) [4-8]. Apart from $\sim 3 \%$ of coding sequences, for an estimate of 20,000-25,000 genes [9], the vast majority of DNA is composed of introns, pseudogenes, duplications, and by at least $50 \%$ repeat sequences (including genes that encode ribosomal components, minisatellite DNA, HERVs, and probably much more) [6,9,10-11]. HERVs are grouped in 26-31 independently acquired families or lineages [12-13]. They are poorly and variably expressed, being highly defective, but a handful of complete proviruses have been described, having the classical genome organization of a retrovirus, i.e., two long terminal repeat (LTR) regions including the gag (capsidic group-associated antigen, coding for the structural matrix, capsid, and nucleocapsid proteins), pol (polymerase/protease, coding for the reverse transcriptase, integrase and protease proteins), and env (envelope, coding for the envelope surface and transmembrane proteins) genes. 
Over time (30-60 million years), all types of mutations occurred, and deleted or mutated versions of inactive retroelements accumulated in the genome. While some transposable elements became inactive, others retained mobility within the genome. Variably inserting in cellular genes, and differentially within allotypes of polymorphic genes, these elements may have determined inheritable, stable gene modifications [6]. Reinfection by replication-competent elements, complementation in trans, retrotransposition in cis, and copying by non-HERV machinery are mechanisms used in most families [12]. In addition, through their LTRs, they may supply new promoters, create new functional exons, alternative splicing products and miRNAs via integration and adaption events, thus contributing to genome plasticity during evolution [14].

The host occasionally takes advantage of the presence of these ancestrally transmitted foreign genomes to serve physiological functions; several possible beneficial effects have been reported [6], including embryo implantation, gene variability, alternative splicing and polyadenylation, mother's immunosuppression to protect the fetal allograft, protection against superinfection by exogenous retroviruses, and involvement in development and/or differentiation. Conversely, HERVs can contribute to the generation of inherited diseases by induction of gene instability and variability, recombination, gene disruption by insertion of HERV sequences, autoimmunity, superantigenic stimulation, production of immunosuppressive factors, cancer through interactions with oncogenes, and activation/inactivation of growth controlling genes $[6,15]$.

Increasing evidence shows links of HERVs with some human diseases, such as male infertility, schizophrenia, and cancer [6,10-11,16]. Several studies reported expression of one or more HERVs in physiological or pathological conditions, in one or more body sites. A key problem is differentiating this expression as the cause or effect of a particular disease. While for various HERV families, only expression of transcripts (and rarely of proteins) [6,15] were reported for the HERV-W family; several independent studies went further, reproducing in vitro and in animal models pathogenic mechanisms of the linked disease.

\section{The HERV-W family: MSRV and syncytin-1}

The most studied HERV family is HERV-W. There have been two milestone discoveries about
HERV-W: the discovery of its founder member, the multiple sclerosis (MS)-associated retrovirus (MSRV, a presumably complete virus, since it is able to form extracellular, infectious virions), released by leptomeningeal cells of MS patients [16-17], and the discovery that syncytin-1 (a protein expressed in human placenta during pregnancy), is encoded by the env gene of ERVWE1, a replication-incompetent HERV-W element located on human chromosome $7 \mathrm{q} 21-22[7,18]$ that has inactivating mutations in the gag and pol genes and is not able to form virus-like particles. In human DNA, there are multiple copies of HERV-W elements; numbers can vary [19-20], but there are about $70 \mathrm{gag}, 100$ pro, and 30 env HERV-Wrelated regions [21-22]; the family is retrotransposably active [12] and generates new recombinant copies in cancer cells [23]. Full-length HERV-Wenv DNA sequences are present on ten human chromosomes (X, $3,4,5,7,12,14,15,17$, and 18), and three additional chromosomes have HERV-W elements containing incomplete HERV-Wenvs spanning $\geq 80 \%$ of the gene [24]. As for their transcription, only the ERVW-1 locus on chromosome 7 is transcribed in a full-length env mRNA that can be translated in a complete protein, syncytin-1; all the other HERV-Wenv genes present in the current version of the human genome either are not transcribed, or originate HERV-Wenv fragments with stop codons and other gene alterations [24].

In the case of syncytin-1, the host subverts the usual situation in which viruses use the cells for their own advantage (replication or persistence); for example, the host uses these ancestrally transmitted viral genomes for pivotal physiological functions during pregnancy. In fact, syncytin-1 is responsible for the fusion of fetal trophoblasts into the placental syncytiotrophoblast layer, which allows for embryo implantation [25]. Other relevant functions that have been hypothesized are the powerful transcriptional regulatory properties of HERV-W LTRs and the possible role of HERV-Wenv expression in pregnancy in suppressing the maternal immune response against the fetal allograft [26-27].

None of the known stably inserted HERV-W elements is replication competent; a study of HERV$\mathrm{W}$ intragenomic spread confirmed that, in the few individuals used for genome-wide analysis, the sequenced HERV-W elements lacked intact open reading frames (ORFs) in all genes within a single copy [22]. Syncytin-1 originates from the ERVWE1 element on chromosome 7; the origin of MSRV is still debated [16]. Sequencing of virionic MSRV genomes 
identified a putative 7,630-nucleotide-long reconstituted retroviral organization with RU5, gag, pol, env, and U3R regions [16]. These sequences belong to the HERV-W family [18], classified in (simple) Gammaretroviruses [13]. MSRV might be either an exogenous HERV-W, or a non-ubiquitous replication-competent member, or a partly defective, non-ubiquitous copy, seldom complemented or recombined within the HERV-W family [24]. Whatever the origin of MSRV, 9\% of healthy Caucasians have circulating virionic MSRV/HERV-W RNA [16].

MSRVenv and syncytin-1 are closely related and difficult to distinguish from each other [26]. A major difference is that only MSRV is found as complete extracellular virus, while syncytin-1 is found only intracellularly or on the plasma membrane [16]. At the protein level, no antibodies discriminating MSRVenv and syncytin-1 are yet available [28]. At the RNA level, MSRVenv and syncytin-1 have 94\% similarity; we observed that MSRVenv sequences have a 12nucleotide insertion in the trans-membrane moiety [29]. Based on this insertion, we developed discriminatory real-time polymerase chain reaction (PCR) assays that can amplify selectively either MSRVenv or syncytin-1 [29].

MSRVenv and syncytin-1 proteins share several biological features, and are potentially pathogenic. They have pro-inflammatory and superantigenic properties, and have been shown to cause neurotoxic effects in vitro and in human or transgenic animal models [30-31]. The proteins may cause neuroinflammation, neurodegeneration, alterations of the immune system, and stress responses; both have been suggested as co-factors triggering the immunopathogenesis of MS. Expression of HERVW/MSRV/syncytin-1 occurs in astrocytes of MS lesions in the brain [31-32], as well as in endothelial and microglial cells [33]. Of note, in the brains of mice with a syncytin construct stereotactically implanted into the corpus callosum, oligodendrocytes (which produce the myelin sheath of the nerve) were shown to be sensitive to syncytin-1-mediated release of redox reactants from astrocytes [31]. Notably, MSRVenv alone was shown to promote experimental allergic encephalomyelitis in mice, the animal model of human MS [34].

\section{MS and HERV-Ws}

MS is a chronic neurological disease that usually begins in early adulthood and leads to progressive neurodegeneration. It causes repeated unpredictable bouts of motor disorders, partial paralysis, sensory abnormalities and visual impairment, with demyelination and gliosis, various degrees of axonal pathology, and episodic or progressive neurological disability [35]. The etiology of MS is complex but still unclear. The immuno-pathogenic phenomena leading to neurodegeneration are thought to be triggered by environmental or viral factors, operating on a predisposing genetic background, and result from an inflammatory process that, among other effects, attacks and destroys oligodendrocytes, the cells that form the myelin sheaths around axons in the brain and spinal cord.

Among the HERVs associated with MS, MSRV has been the most intensely studied, followed by syncytin-1. However, due to the sequence similarities, effective distinction between the two elements by discriminatory quantitative real-time reverse transcription (RT)-PCR assays was carried out only by our group [24,28-29,36] and later on, with the same assay, by Perron et al. [37]. With the aid of these realtime PCR assays, which can amplify selectively either MSRVenv or syncytin-1 [29], we found that the DNA of MS patients had increased MSRVenv copies (sixfold mean increase, $\mathrm{p}=0.02$ ), while the syncytin1 -specific assay gave almost identical mean values for both patients and controls [29].

To date, the presence of extracellular MSRV particles in MS patients and the expression of MSRVenv and syncytin-1 transcripts have been detected independently in the blood and the brain by several groups, and found to be increased in both healthy and pathological controls [6,15-16], and shown in Figure 1 for our Sardinian cohort.

Our group has studied MSRV/syncytin-1 expression in MS patients since 1998, initially because the Italian island of Sardinia has the highest MS prevalence worldwide (more than 230/100,000 inhabitants) [38]. In the Sardinian cohort, circulating MSRV was detected in $100 \%$ of patients with active MS (but in 33\% of MS patients in remitting phase, as reported in Figure 2); moreover, the presence of MSRV particles in spinal fluid was found to parallel temporal and clinical progression of the disease [39].

In the brain, we found that the levels of MSRV/HERV-Wenv transcripts are similar in samples from normal controls (autopsied tissues from subjects with non-neurological diseases), and from pathological controls (tissues from patients with non-MS neurological diseases), without significant differences between white and grey matter. 
Figure 1. Extracellular MSRV in the blood of MS patients and of healthy and pathological controls

(A)

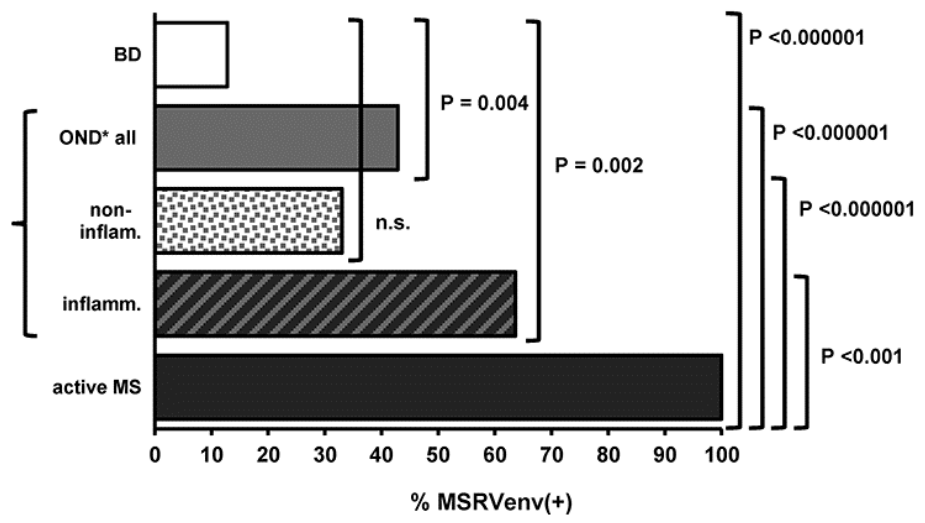

(B)

MSRVpol, nested RT-PCR

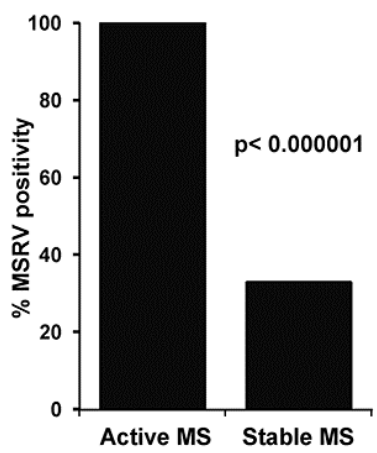

MSRVenv, real time RT-PCR,

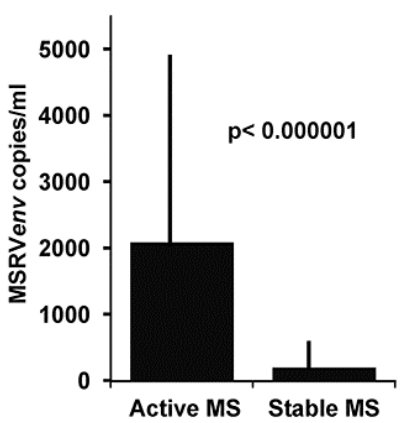

(A) Percentage of MSRV-positivity in healthy blood donors (BD), control patients with other neurological diseases (OND) with or without inflammation, and MS patients in active phase of disease. (B) Percentage of MSRV release in the blood of MS patients during stable and active MS disease, as detected by RT-PCR assays detecting the pol and env genes (left and right panels, respectively). For details, see [39].

Figure 2. Extracellular MSRV in the cerebrospinal fluids of MS patients and of pathological controls

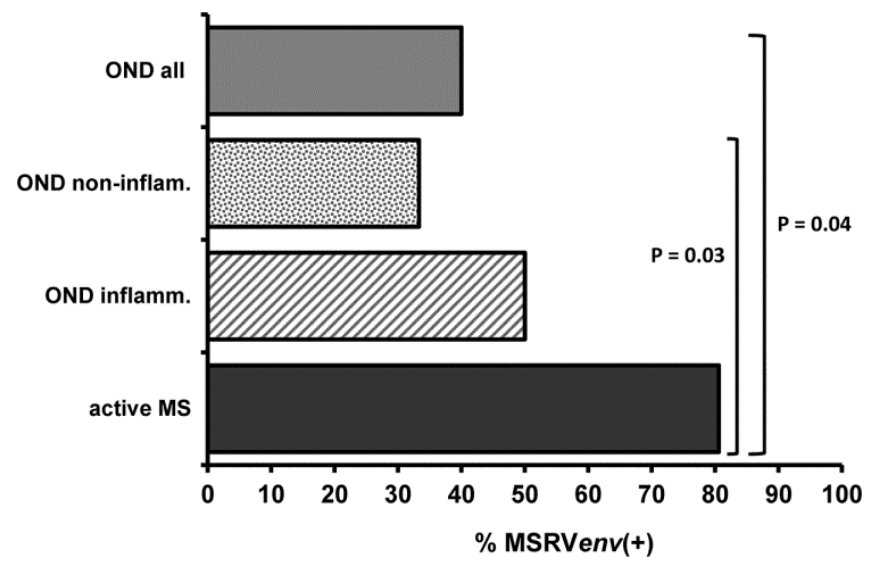

OND: control patients with other neurological diseases, with or without inflammation, and MS patients in active phase of disease, as detected by RT-PCR assay [39]. 
In brain lesions from MS patients, the levels of MSRV/HERV-W transcripts were increased by 20 - to 25 -fold ( $\mathrm{p}=0.014$ versus healthy controls; $\mathrm{p}=0.006$ versus pathological controls). By immunohistochemistry, no HERV-Wenv protein was detected in control brains, whereas it was highly expressed within MS plaques and correlated with the extent of active demyelination and inflammation. At the lesion edge, HERV-W immunostaining was detected on cells resembling both astrocytes and microglia, while in plaque centers, the HERV-W signal was mostly localized on hypertrophic astrocytes [29].

A large multicentric study of MS patients and neurological and healthy controls from different European areas showed that MSRV presence and load in blood and spinal fluid was significantly associated with MS in all ethnic groups $(\mathrm{p}<0.05$ compared to neurological controls and $<0.001$ compared to healthy controls) [40]. MRSV presence/load in MS patients strikingly paralleled disease behavior; we detected direct parallelisms between MSRV positivity/load (in blood, spinal fluid, and brain samples) and MS temporal and clinical stages, as well as active/remission phases (Figure 2). MSRV positivity of spinal fluids increased with MS duration [39], and its presence in early MS was related to worse prognosis in the next ten years. A blind observational study of early MS patients starting in comparable conditions (but differing in MSRV positivity in the spinal fluid) confirmed that the presence of MSRV in the cerebrospinal fluid of early MS patients was associated with a significantly greater rate of disability and disease progression, since after three [41], six [42], and ten years [43], mean disability, annual relapse rate, therapy requirement, and progression to secondary-progressive MS were significantly higher in patients starting with MSRV-positive spinal fluids (Figure 3). Hence, we proposed that evaluation of plasmatic MSRV could be considered the first prognostic marker for the individual patient to monitor disease progression. Our proposal was reinforced by the study of patients with optic neuritis, a disease frequently prodromic to MS; in the blood and in the spinal fluid of these patients, MSRV positivity was significantly higher than in that of pathological controls, and the conversion to full-blown MS in the next 20 months occurred only among MSRV-positive patients [44].

A positive feedback loop on MSRV production was observed in cultured peripheral blood mononuclear cells (PBMC) from MSRV-positive individuals [45], where MSRV release was upregulated by pro-inflammatory cytokines such as TNF $\alpha$, IL- 6 , and interferon- $\gamma$, while it was inhibited by the beneficial interferon- $\beta$ (Figure 4 ). These proinflammatory cytokines, in turn, are overproduced in response to MSRV/HERV-Wenv by cells from MS

Figure 3. Follow-up for ten years of patients with early multiple sclerosis

\section{(A)}

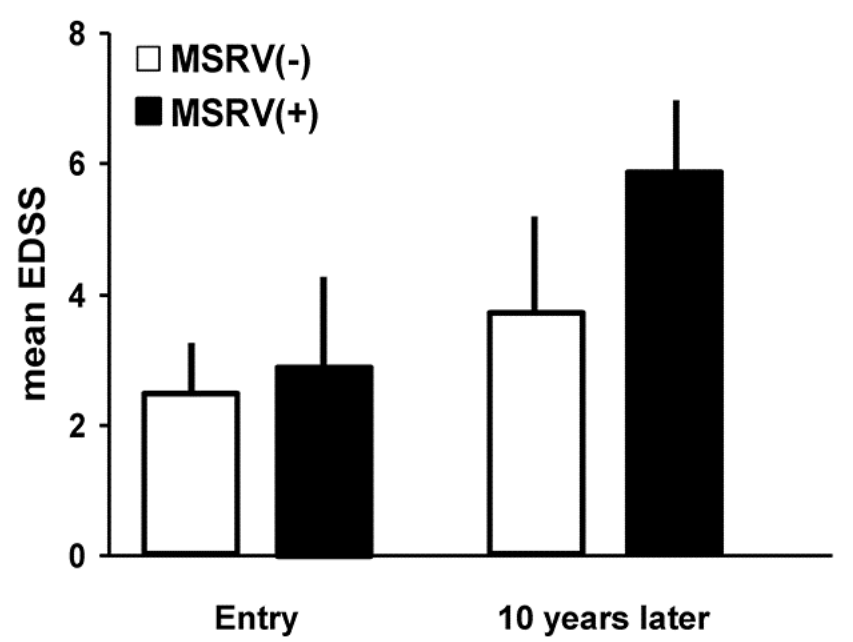

(B)

\begin{tabular}{|c|c|c|c|c|}
\hline & & & & RV(+) \\
\hline & RMS & PMS & RRMS & SPMS \\
\hline Onset & 8 & 0 & 18 & 0 \\
\hline $10 \mathrm{yrs}$ & 8 & 0 & 12 & 8 \\
\hline$\%$ SP & & $\%$ & & $44 \%$ \\
\hline
\end{tabular}

Blind observational study of early MS patients starting from similar conditions, but differing in MSRV presence in the spinal fluid. (A) Early MSRV detection in the spinal fluid of MS patients is associated with higher rate of disability, as measured by Kurtzke's expanded disability status scale (EDSS) score on Y-axis. MSRV-positive patients, after 10 years, (black bars) have significantly higher disability as compared with their counterparts that, at time zero, were MSRV-negative (white bars; t-test, $\mathrm{p}<0.004$ ) and with the average EDSS at study entry (t-test, $\mathrm{p}<0.001$ ). (B) The progression of the disease toward the secondary-progressive form of MS (SPMS) after 10 years occurs only on those patients that were MSRV-negative at onset. For details, see [42]. 
patients, and correlate with MS severity [46], thus providing a pathogenic amplification loop.

Next, we verified whether MSRV expression varies in MS patients during therapy. A longitudinal evaluation of MS patients during efficacious therapy with interferon- $\beta$ revealed that MSRV viremia fell rapidly below detection limits (the earliest effect was detected 48 hours after the first drug inoculation); notably, a patient, after initial clinical and virological benefit, had MSRV rescue, preceding strong disease progression and therapy failure [47]. Our findings were confirmed by an independent follow-up study that observed significant decreases in anti-HERVWenv and anti-HERV-Henv antibody reactivity as a consequence of interferon- $\beta$ therapy, closely linked to efficacy of therapy/low disease activity [48]. In another longitudinal study, we showed that natalizumab (a humanized monoclonal antibody indicated for MS patients who have not responded to conventional MS treatments) strongly reduced MSRV/syncytin-1/HERV-W expression, which parallels the clinical benefit of the therapy [49].

All the above data $[32,39-45,47,49]$ and data from other groups $[37,48]$ strengthen our hypothesis that the evaluation of MSRV/syncytin/HERV-W expression/release could be considered the first prognostic marker for the individual patient to monitor disease progression and therapy outcome.

\section{The viral trigger of MS pathogenesis and the dual virus hypothesis}

Several viruses have been proposed as co-factors of MS [50-51]. Among them, there were some members of the family of Herpesviridae, such as the human herpesvirus 6 (HHV-6) [52], and the EpsteinBarr virus (EBV) [53], along with HERVW/MSRV/syncytin-1 [16,50,54].

With respect to HHV-6, meta-analyses provided some support for a link between HHV-6 and MS, but none showed causative relationships, and findings across the field are non-uniform [52]. Since we found significant increases of the expression of HERV-W in

Figure 4. MSRV release by PBMC from MSRV-positive donors exposed to cytokines
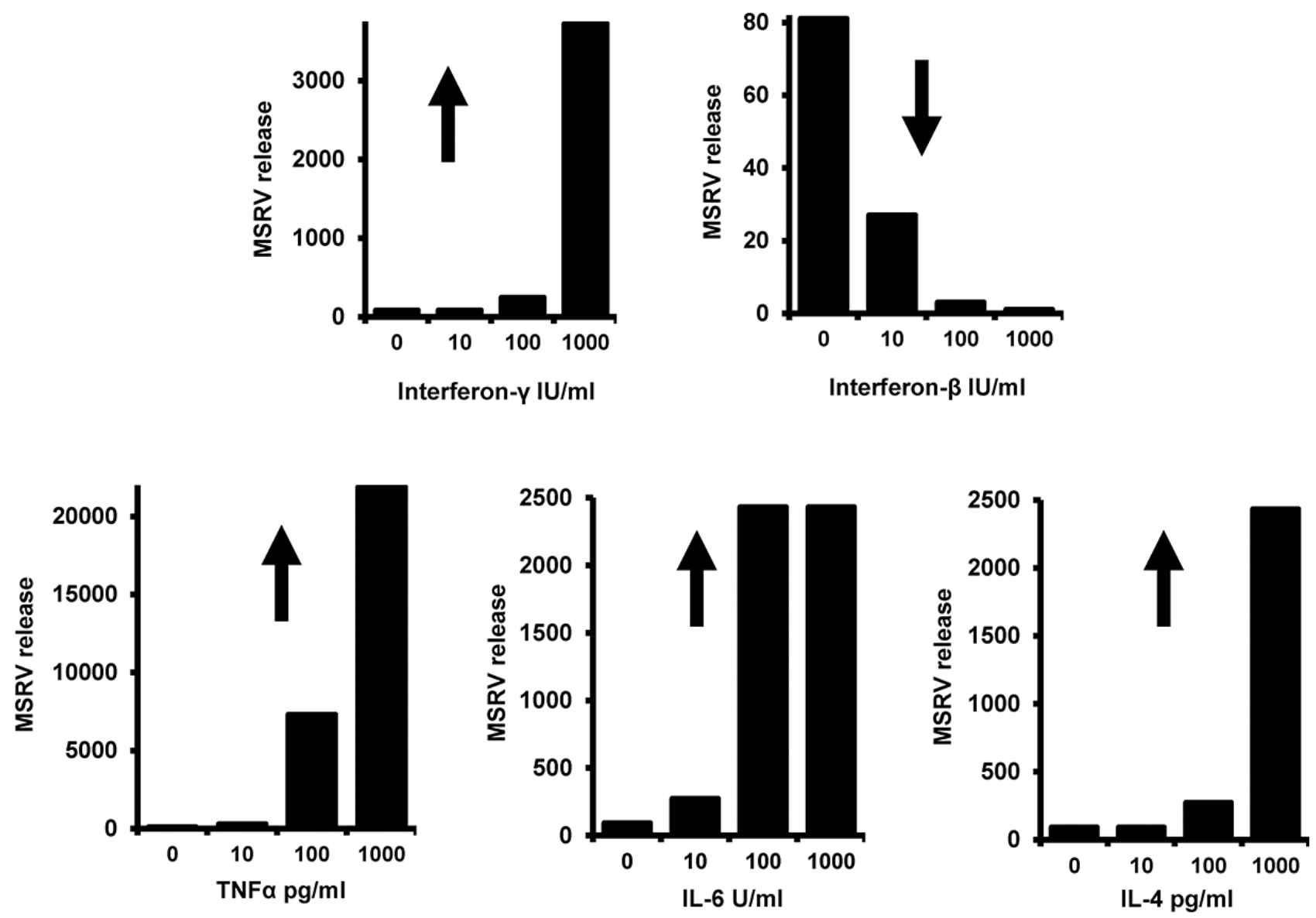

MSRV release in culture by mitogen-activated PBMC from MSRV-positive healthy donors that were exposed for four days to graded amounts of interferon- $\gamma(\mathrm{A})$, interferon- $\beta$ (B), TNF $\alpha$ (C), IL-6 (D), and IL-4 (E). For details, see [44]. 
MS patients, we evaluated the expression of both HERV-Ws and HHV-6 within the same samples of brain and blood cells of MS patients and controls, as the mere detection of HHV-6 would not imply viral activity. This is particularly important for HHV-6 studies, as the vast majority of reports have detected virus DNA, and the need for studies to examine antigen and virus mRNA expression in MS and control brains to delineate the relationship between latent and active virus and MS has been emphasized [52]. By evaluating two different transcripts for each virus (MSRV/HERV-W env and pol, HHV-6 U94/rep, which maintains the latent state, and DNA-pol, indicative of actively replicating virus), we found that MSRV/HERV-W was expressed actively in human brains and activated strongly in MS patients (up to the protein level), while there were no significant differences between these MS patients and controls for HHV-6 presence/replication at the brain or PBMC level [32].

More consistent studies for potential virus involvement in MS exist for EBV [53], as the risk of developing MS is higher among individuals infected with EBV, especially if the EBV infection occurs in late adolescence or adulthood, when the infection is symptomatic. A meta-analysis showed an association between the appearance of anti-EBV antibodies and the onset of MS 5-20 years later [55]; another paper reported that the relative risk of MS for a past history of infectious mononucleosis (IM) is 2.17 [56]. The MS-EBV link remains unclear and seems to be indirect, since no studies have found evidence of EBV expression specific to MS, and currently there is no consensus in establishing whether there is a presence of EBV-infected cells in the central nervous system (CNS) of MS patients [57]. Therefore, it remains to be determined whether EBV continues to play a role after disease initiation $[24,36]$.

In search of EBV/HERV-W/MSRV/syncytin-1 links, we studied in vitro the expression of HERVW/MSRV/syncytin-1, with or without exposure to EBV or to EBV glycoprotein350 (EBVgp350, the major envelope protein), on PBMC from healthy volunteers and MS patients, and on astrocytes, by discriminatory $e n v$-specific real-time RT-PCR assays and by flow cytometry [24]. We verified whether HERV-W might be activated also in vivo, in hospitalized young adults with IM symptoms, and in healthy controls that were either EBV negative or latently EBV infected with or without high titers of anti-EBNA-1 IgG [24,36]. Briefly, the results showed that in vitro EBV activates the potentially immunopathogenic and neuropathogenic HERVW/MSRV/syncytin-1 in cells deriving from the blood and brain. In vivo HERV-W/MSRV activation is higher in blood cells of IM patients and in healthy controls with high anti-EBNA-1 IgG titers. Thus, the data indicate that the two main links between EBV and MS (IM and high anti-EBNA-1-IgG titers) are paralleled by activation of the potentially neuropathogenic HERV-W/MSRV. Considering our data and those of the literature on MS pathogenesis, we postulate the possibility that EBV is an initial trigger of future MS, years later, and that MSRV has a direct role as effector of neuropathogenesis before and during MS [24,36].

\section{MSRV/HERV-W/syncytin-1 retroviruses and neurodegeneration}

endogenous HIV-related

The central nervous system (CNS) is a major target for the human immunodeficiency virus (HIV) [58-60]. HIV neuroinvasion in vivo occurs within the first month [61], and this early compartmentalization suggests a CNS reservoir for local virus production [62]. The neurodegenerative disease (neuroAIDS) progresses during chronic infection, with neurocognitive, behavioral, and motor impairments that can lead to full-blown HIV-associated dementia [58]. The mechanisms by which HIV causes neurodegeneration are not fully elucidated, and include both host and viral mechanisms [28,63]. Additional relevant contributions to neuroAIDS are caused by reactivation of bystander latent/persistent viruses with neurotropic potential such as EBV, cytomegalovirus, and JC polyomavirus, which can result in life-threatening CNS infections in immunocompromised hosts [28].

Since $10 \%$ of healthy Caucasian release MSRV in the blood [16], we wondered whether MSRV and syncytin- 1 are activated by HIV and thus whether their neuropathogenic and immunopathogenic properties could contribute to HIV-related neurodegeneration [28]. In fact, rather than HIV itself, as the virus production is too low to explain the extensive CNS disease often observed, mechanisms of amplification of toxicity and inflammation in response to HIV infection were suggested as a major cause of neurological disease [64]. To this end, we exposed to HIV, or to its transactivator of transcription (Tat) protein, monocytes/macrophages and astrocytes, i.e., the cells that are thought to be more relevant for HIVrelated neurodegeneration [28]. 
The results showed that HIV infection and exposure to Tat increase the levels of MSRVenv mRNAs and HERV-Wenv proteins in astrocytes and in blood cells. In monocyte-macrophages, Tat also induces high levels of CCR2, CD16, and Toll-like receptor 4 (TLR4) molecules. Interestingly, syncytin-1 response to Tat depends on the cell context: in monocytes, Tat stimulates MSRVenv and inhibits syncytin-1, while in differentiated macrophages and primary astrocytes, Tat stimulates both elements [28].

Experiments were performed to give insights on the mechanisms by which Tat regulates MSRV and syncytin-1. It is known that $\mathrm{TNF} \alpha$ is the most abundant proinflammatory cytokine in the brains of neuroAIDS patients; in this context of neurodegeneration, MSRV and syncytin-1 stimulation by the induced TNF $\alpha$ seemed likely. We found that Tat internalization by exposed cells is not required [28]. In primary astrocytes, Tat stimulates MSRV and syncitin-1 through interaction with TLR4 and induction of TNF $\alpha$, as reported on Figure 5. The indirect mechanism by which Tat activates HERV-Ws by $\mathrm{TNF} \alpha$ induction could be a new property of this pleiotropic protein. The in vivo consequence of the study could be that, through increase of CD16 and CCR2, Tat promotes neuroinvasion by HIV-infected monocytes/macrophages, but also by HERV-Ws, with their neuropathogenic potential. Within the brain, Tatinduced TNF $\alpha$ could induce high levels of HERV-Ws, in both macrophages and astrocytes, also without HIV replication.

Figure 5. Cartoon of the proposed mechanism of MSRV and syncytin-1 activation by HIV Tat in human astrocytes

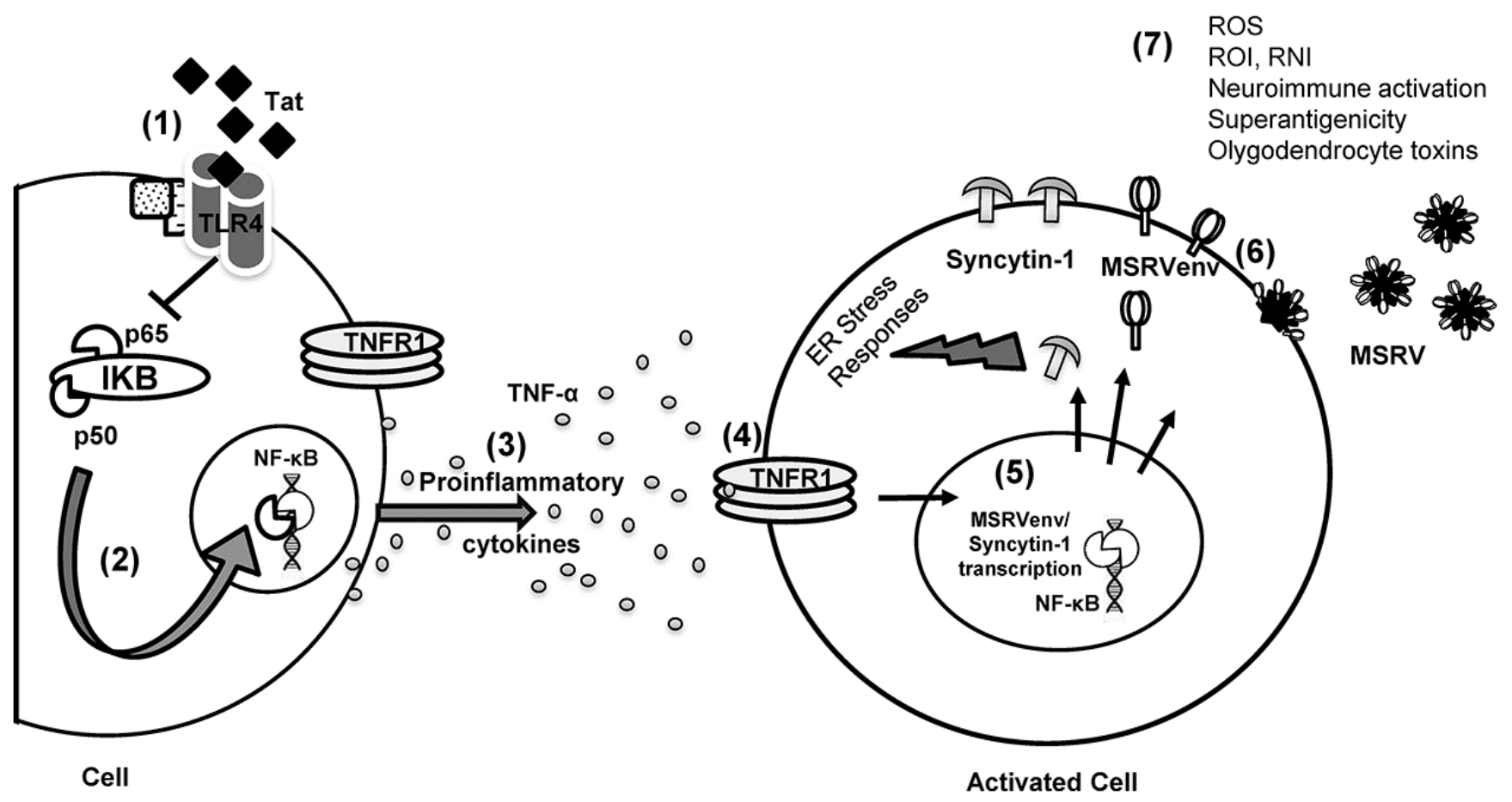

(1) HIV Tat bind<s the TLR4-MD2 complex on the membrane; this activates TNF $\alpha$ transcription, by two main pathways (NF- $\mathrm{kB}$ through the TRIFdependent pathway and/or through a MyD88-dependent pathway). (2) The two pathways lead to proteasomal degradation of I $\mathrm{kBs}$ (inhibitor of $\kappa B$ ), allowing NF- $\mathrm{KB}$ translocation into the nucleus. (3) Consequent innate immune response, with production of TNF $\alpha$ and others proinflammatory cytokines. (4) The released TNF $\alpha$ binds to TNF receptor (TNFR), to initiate a second run of NF- $\mathrm{kB}$ activation in a autocrine (not shown) and paracrine manner. (5) The signalling pathway downstream TNFR leads to nuclear translocation of NF- $\kappa \mathrm{B}$, where it binds to the promoters of syncytin1 and MSRV, hence (6) activating the production/release of the corresponding env proteins and MSRV particles. (7) Cytoplasmatic accumulation of env proteins induces endoplasmic reticulum (ER) stress responses; extracellular or transmembrane env proteins can exert their properties: fusogenicity, capacity to activate the neuroimmune system, to damage oligodendrocytes and interfere with myelin regeneration, etc. For details, see [28].

Abbreviations: TLR4: toll like receptor 4; TLR4-CD14-MD2: lipopolysaccharide receptor complex, consisting of two interacting receptors (CD14 and TLR4) and an associated protein (MD-2); TNFa: tumor necrosis factor alpha; NFאB: nuclear factor $\kappa B$; TNFR1: tumor necrosis factor receptor 1; ER: endoplasmic reticulum; ROI: reactive oxygen intermediates; RNI: reactive nitrogen intermediates.

*** Other receptor/endocytic-mediated Tat pathways ( $\alpha 5 \beta 1 / \alpha-v \beta 3$ integrins; CD26; LRP; HSPGs). 


\section{Concluding remarks}

HERVs constitute a significant part of our genome, and were passed successfully over 10-60 million years in the genome of our ancestors. Mutual adaptation must have occurred, and in some cases, the host turned to his own use some of these foreign genomes to serve physiological functions, as in the case of HERV-W/syncytin-1, a mediator of the formation of the syncytiotrophoblast layer during early pregnancy. Over time, some retroelements may have originated positive genetic variability, but also inheritable or inducible diseases.

In patients with MS, HERV-W/MSRV can be considered a biomarker for MS behavior and therapy outcome. As for MS pathogenesis, we postulate the possibility that EBV is an initial trigger of future MS, and that MSRV has a direct role of effector of neuropathogenesis during MS. In HIV-infected patients, HERV-W/MSR/syncytin-1 activation by HIV Tat could contribute to HIV-related neurodegeneration.

A key problem when evaluating the expression is differentiating whether it is the cause or effect of a particular disease or physiological condition.

The links of some HERVs to human diseases are increasingly observed, particularly those characterized by multifactorial etiology and/or dysregulated immune functions, and there is hope for identifying new targets for innovative therapeutic interventions.

\section{Acknowledgements}

This work was supported in part by CARITRO 2013 (AD) and RAS 2013. EU was funded by a grant from CARITRO 2013.

\section{References}

1. Griffiths DJ (2001) Endogenous retroviruses in the human genome sequence. Genome Biol 2: reviews1017reviews1017.5.

2. Tarlinton RE, Meers J, Young PR (2006) Retroviral invasion of the koala genome. Nature 442: 79-81.

3. Ishida Y, Zhao K, Greenwood AD, Roca AL (2015) Proliferation of endogenous retroviruses in the early stages of a host germ line invasion. Mol Biol Evol 32: 109-120.

4. Hino S, Tronick SR, Heberling RL, Kalter SS, Hellman A, Aaronson SA (1977) Endogenous New World primate retrovirus: interspecies antigenic determinants shared with the major structural protein of type-D RNA viruses of Old World monkeys. Proc Natl Acad Sci U S A 74: 5734-5738.

5. Keshet E, Temin HM (1977) Nucleotide sequences derived from pheasant DNA in the genome of recombinant avian leukosis viruses with subgroup F specificity. J Virol 24: 505513.

6. Dolei A (2006) Endogenous retroviruses and human disease. Expert Rev Clin Immunol 2: 149-167.

7. Tristem M (2000) Identification and characterisation of novel human endogenous retrovirus families by phylogenetic screening of the human genome mapping project database. J Virol 74: 3715-3730.

8. Pennisi E (2012) Genomics. ENCODE project writes eulogy for junk DNA. Science 337: 1159-1161.

9. International Human Genome Sequencing Consortium (2004) Finishing the euchromatic sequence of the human genome. Nature 431: 931-945.

10. Nelson PN, Hooley P, Roden D, Davari Ejtehadi H, Rylance P, Warren P, Martin J, Murray PG (2004) Molecular Immunology Research Group. Human endogenous retroviruses:transposable elements with potential? Clin Exp Immunol 138: 1-9.

11. Kim YJ, Lee J, Han K (2012) Transposable Elements: No More 'Junk DNA'. Genomics Inform 10: 226-233.

12. Belshaw R, Katzourakis A, Paces J, Burt A, Tristem M (2005) High copy number in human endogenous retrovirus families is associated with copying mechanisms in addition to reinfection. Mol Biol Evol 22: 814-817.

13. Weiss RA (2006) The discovery of endogenous retroviruses. Retrovirology 3: 67.

14. Kim HS (2012) Genomic impact, chromosomal distribution and transcriptional regulation of HERV elements. Mol Cells 33: $539-544$

15. Antony JM, Deslauriers AM, Bhat RK, Ellestad KK, Power C (2011) Human endogenous retroviruses and multiple sclerosis: innocent bystanders or disease determinants? Biochim Biophys Acta 1812: 162-176.

16. Dolei A, Perron H (2009) The multiple sclerosis-associated retrovirus and its HERV-W endogenous family: a biological interface between virology, genetics, and immunology in human physiology and disease. J Neurovirol 1: 4-13.

17. Perron H, Geny C, Laurent A, Mouriquand C, Pellat J, Perret J, Seigneurin JM (1989) Leptomeningeal cell line from multiple sclerosis with reverse transcriptase activity and viral particles. Res Virol 140: 551-561.

18. Blond JL, Beseme F, Duret L, Bouton O, Bedin F, Perron H, Mandrand B, Mallet F (1999) Molecular characterisation and placental expression of HERV-W, a new human endogenous retrovirus family. J Virol 73: 1175-1185.

19. Mirsattari SM, Johnston JB, McKenna R, Del Bigio MR, Orr P, Ross RT, Power C (2001) Aboriginals with multiple 
sclerosis: HLA types and predominance of neuromyelitis optica. Neurology 56: 317-323.

20. Zawada M, Liweñ I,Pernak M, Januszkiewicz-Lewandowska D, Nowicka-Kujawska K, Rembowska J, Lewandowski K, Hertmanowska H, Wender M, Nowak J (2003) MSRV pol sequence copy number as a potential marker of multiple sclerosis. Pol J Pharmacol 55: 869-875.

21. Voisset C, Bouton O, Bedin F, Duret L, Mandrand B, Mallet F, Paranhos-Baccala G (2000) Chromosomal distribution and coding capacity of the human endogenous retrovirus HERVW family. AIDS Res Hum Retroviruses 16: 731-740.

22. Kim HS, Kim DS, Huh JW, Ahn K, Yi JM, Lee JR, Hirai H (2008) Molecular characterization of the HERV-W env gene in humans and primates: expression, FISH, phylogeny, and evolution. Mol Cells 26: 53-60.

23. Yi JM, Kim HM, Kim HS (2004) Expression of human endogenous retrovirus HERV-W family in various human tissues and cancer cells. J Gen Virol 85: 1203-1210.

24. Mameli G, Poddighe L, Mei A, Uleri E, Sotgiu S, Serra C, Manetti R, Dolei A (2012) Expression and activation by Epstein Barr virus of human endogenous retroviruses-W in blood cells and astrocytes: inference for multiple sclerosis. PLoS One 7: e44991.

25. Mi S, Lee X, Li X, Veldman GM, Finnerty H, Racie L, LaVallie E, Tang XY, Edouard P, Howes S, Keith JC Jr, McCoy JM (2000) Syncytin is a captive retroviral envelope protein involved in human placental morphogenesis. Nature 403: 785-789.

26. Dolei A (2005) MSRV/HERV-W/syncytin and its linkage to multiple sclerosis: the usability and the hazard of a human endogenous retrovirus. J Neurovirol 11: 232-235.

27. Lokossou AG, Toudic C, Barbeau B (2014) Implication of human endogenous retrovirus envelope proteins in placental functions. Viruses 6: 4609-4627.

28. Uleri E, Mei A, Mameli G, Poddighe L, Serra C, Dolei A (2014) HIV Tat acts on endogenous retroviruses of the W family and this occurs via Toll-like receptor 4: inference for neuroAIDS. AIDS 28: 2659-2670.

29. Mameli G, Poddighe L, Astone V, Delogu G, Arru G, Sotgiu S, Serra C, Dolei A (2009) Novel reliable real-time PCR for differential detection of MSRVenv and syncytin-1 in RNA and DNA from patients with multiple sclerosis. J Virol Methods 161: 98-106.

30. Firouzi R, Rolland A, Michel M, Jouvin-Marche E, Hauw JJ, Malcus-Vocanson C, Lazarini F, Gebuhrer L, Seigneurin JM, Touraine JL, Sanhadji K, Marche PN, Perron H (2003) Multiple sclerosis-associated retrovirus particles cause $\mathrm{T}$ lymphocyte-dependent death with brain hemorrhage in humanized SCID mice model. J Neurovirol 9: 79-93.

31. Antony JM, Van Marle G, Opii W, Butterfield DA, Mallet F, Yong VW, Wallace JL, Deacon RM, Warren K, Power C (2004) Human endogenous retrovirus glycoprotein-mediated induction of redox reactants causes oligodendrocyte death and demyelination. Nat Neurosci 7: 1088-1095.

32. Mameli G, Astone V, Arru G, Marconi S, Lovato L, Serra C, Sotgiu S, Bonetti B, Dolei A (2007) Brains and peripheral blood mononuclear cells of multiple sclerosis (MS) patients hyperexpress MS-associated retrovirus/HERV-W endogenous retrovirus, but not Human herpesvirus 6. J Gen Virol 88: 264274.

33. Perron H, Lazarini F, Ruprecht K, Pechoux-Longin C, Seilhean D, Sazdovitch V, Créange A, Battail-Poirot N, Sibaï G, Santoro L, Jolivet M, Darlix JL, Rieckmann P, Arzberger
T, Hauw JJ, Lassmann H (2005) Human endogenous retrovirus (HERV)-W ENV and GAG proteins: physiological expression in human brain and pathophysiological modulation in multiple sclerosis lesions. J Neurovirol 11: 23-33.

34. Perron H, Dougier-Reynaud HL, Lomparski C, Popa I, Firouzi R, Bertrand JB, Marusic S, Portoukalian J, JouvinMarche E, Villiers CL, Touraine JL, Marche PN (2013) Human endogenous retrovirus protein activates innate immunity and promotes experimental allergic encephalomyelitis in mice. PLoS One 8: e80128.

35. McQualter JL, Bernard CC (2007) Multiple sclerosis: a battle between destruction and repair. J Neurochem 100: 295-306.

36. Mameli G, Madeddu G, Mei A, Uleri E, Poddighe L, Delogu LG, Maida I, Babudieri S, Serra C, Manetti R, Mura MS, Dolei A (2013) Activation of MSRV-type endogenous retroviruses during infectious mononucleosis and EpsteinBarr virus latency: the missing link with multiple sclerosis? PLoS One 8: e78474.

37. Perron H, Germi R, Bernard C, Garcia-Montojo M, Deluen C, Farinelli L, Faucard R, Veas F, Stefas I, Fabriek BO, VanHorssen J, Van-der-Valk P, Gerdil C, Mancuso R, Saresella M, Clerici M, Marcel S, Creange A, Cavaretta R, Caputo D, Arru G, Morand P, Lang AB, Sotgiu S, Ruprecht K, Rieckmann P, Villoslada P, Chofflon M, Boucraut J, Pelletier J, Hartung HP (2012) Human endogenous retrovirus type W envelope expression in blood and brain cells provides new insights into multiple sclerosis disease. Mult Scler 18: 17211736.

38. Sardu C, Cocco E, Mereu A, Massa R, Cuccu A, Marrosu MG, Contu P (2012) Population based study of 12 autoimmune diseases in Sardinia, Italy: prevalence and comorbidity. PLoS One 7: e32487.

39. Dolei A, Serra C, Mameli G, Pugliatti M, Sechi G, Cirotto MC, Rosati G, Sotgiu S (2002) Multiple sclerosis-associated retrovirus (MSRV) in Sardinian MS patients. Neurology 58: 471-473.

40. Arru G, Mameli G, Astone V, Serra C, Huang YM, Link H, Fainardi E, Castellazzi M, Granieri E, Fernandez M, Villoslada P, Fois ML, Sanna A, Rosati G, Dolei A, Sotgiu S (2007) Multiple Sclerosis and HERV-W/MSRV: A Multicentric Study. Int J Biomed Sci 3: 292-297.

41. Sotgiu S, Serra C, Mameli G, Pugliatti M, Rosati G, Arru G, Dolei A (2002) Multiple sclerosis (MS)-associated retrovirus and MS prognosis: an observational study. Neurology 59: 1071-1073.

42. Sotgiu S, Mameli G, Serra C, Zarbo IR, Arru G, Dolei A (2010) Multiple sclerosis-associated retrovirus and progressive disability of multiple sclerosis. Mult Scler 16: 1248-1251.

43. Sotgiu S, Arru G, Mameli G, Serra C, Pugliatti M, Rosati G, Dolei A (2006). MSRV in early multiple sclerosis: a six-year follow-up of a Sardinian cohort. Mult Scler 12: 698-703.

44. Sotgiu S, Arru G, Söderström M, Mameli G, Serra C, Dolei A (2006) MSRV and optic neuritis. Mult Scler 12: 357-359.

45. Serra C, Mameli G, Arru G, Sotgiu S, Rosati G, Dolei A (2003) In vitro modulation of the multiple sclerosis (MS)associated retrovirus (MSRV) by cytokines: implications for MS pathogenesis. J Neurovirol 9: 637-643.

46. Rolland A, Jouvin-Marche E, Saresella M, Ferrante P, Cavaretta R, Creange A, Marche P, Perron H (2005) Correlation between disease severity and in vitro cytokine production mediated by MSRV (multiple sclerosis associated 
retroviral element) envelope protein in patients with multiple sclerosis. J Neuroimmunol 160: 195-203.

47. Mameli G, Serra C, Astone V, Castellazzi M, Poddighe L, Fainardi E, Neri W, Granieri E, Dolei A (2008) Inhibition of multiple-sclerosis-associated retrovirus as biomarker of interferon therapy. J Neurovirol 14: 73-77.

48. Petersen T, Møller-Larsen A, Thiel S, Brudek T, Hansen TK, Christensen T (2009) Effects of interferon-beta therapy on innate and adaptive immune responses to the human endogenous retroviruses HERV-H and HERV-W, cytokine production, and the lectin complement activation pathway in multiple sclerosis. J Neuroimmunol 215: 108-116.

49. Arru G, Leoni S, Pugliatti M, Mei A, Serra C, Delogu LG, Manetti R, Dolei A, Sotgiu S, Mameli G (2014) Natalizumab inhibits the expression of human endogenous retroviruses of the $\mathrm{W}$ family in multiple sclerosis patients: a longitudinal cohort study. Mult Scler 20: 174-182.

50. Cusick MF, Libbey JE, Fujinami RS (2013) Multiple sclerosis: autoimmunity and viruses. Curr Opin Rheumatol 25: 496-501.

51. Libbey JE, Cusick MF, Fujinami RS (2014) Role of pathogens in multiple sclerosis. Int Rev Immunol 33: 266283.

52. Leibovitch EC, Jacobson S (2014) Evidence linking HHV-6 with multiple sclerosis: an update. Curr Opin Virol 9: 127133.

53. Ascherio A (2013) Environmental factors in multiple sclerosis. Expert Rev Neurother 13 Suppl 12: 3-9.

54. Dolei A, Garson JA, Arru G, Clerici M, Germi R, Marche PN, Perron H (2014) Multiple sclerosis-associated retrovirus and related human endogenous retrovirus-W in patients with multiple sclerosis. J Neuroimmunol 266: 87-88.

55. Santiago O, Gutierrez J, Sorlozano A, De Dios Luna J, Villegas E, Fernandez O (2010) Relation between EpsteinBarr virus and multiple sclerosis: analytic study of scientific production. Eur J Clin Microbiol Infect Dis 29: 857-866.

56. Handel AE, Williamson AJ, Disanto G, Handunnetthi L, Giovannoni G, Ramagopalan SV (2010) An updated metaanalysis of risk of multiple sclerosis following infectious mononucleosis. PLoS One 5: e12496.
57. Pakpoor J, Giovannoni G, Ramagopalan SV (2013) EpsteinBarr virus and multiple sclerosis: association or causation? Expert Rev Neurother 13: 287-297.

58. Shapshak P, Kangueane P, Fujimura RK, Commins D, Chiappelli F, Singer E, Levine AJ, Minagar A, Novembre FJ, Somboonwit C, Nath A, Sinnott JT (2011) Editorial neuroAIDS review. AIDS 25: 123-141.

59. Hong S, Banks WA (2015) Role of the immune system in HIV-associated neuroinflammation and neurocognitive implications. Brain Behav Immun 45: 1-12.

60. Gray LR, Roche M, Flynn JK, Wesselingh SL, Gorry PR, Churchill MJ (2014) Is the central nervous system a reservoir of HIV-1? Curr Opin HIV AIDS 9: 552-558.

61. Davis LE, Hjelle BL, Miller VE, Palmer DL, Llewellyn AL, Merlin TL, Young SA, Mills RG, Wachsman W, Wiley CA (1992) Early viral brain invasion in iatrogenic human immunodeficiency virus infection. Neurology 42: 1736-1739.

62. Spudich S (2013) HIV and neurocognitive dysfunction. Curr HIV/AIDS Rep 10: 235-243.

63. Desplats P, Dumaop W, Smith D, Adame A, Everall I, Letendre S, Letendre S, Ellis R, Cherner M, Grant I, Masliah E (2013) Molecular and pathologic insights from latent HIV-1 infection in the human brain. Neurology 80: 1415-1423.

64. Eugenin EA, Berman JW (2013) Cytochrome C dysregulation induced by HIV infection of astrocytes results in bystander apoptosis of uninfected astrocytes by an IP(3) and calciumdependent mechanism. J Neurochem 127: 644-651.

\section{Corresponding author}

Antonina Dolei

Department of Biomedical Sciences, University of Sassari

Viale San Pietro 43B, 07100

Sassari, Italy

Phone: +393204299685

Fax: +39079212345

Email: doleivir@uniss.it

Conflict of interests: No conflict of interests is declared. 